\title{
LES DISPOSITIFS PARTICIPATIFS : UNE MODALITÉ DE L'APPRENTISSAGE ORGANISATIONNEL
}

\author{
Christel CHRISTOPHE ${ }^{1}$
}

Notre communication traite de la participation sous l'angle de l'étude des dispositifs participatifs proposés par une organisation dédiée à l'accompagnement d'entrepreneurs sociaux. Fondée sur les résultats d'une ethnographie, nous proposons une réflexion sur la manière dont ces dispositifs participatifs contribuent à l'apprentissage organisationnel en développant une approche inspirée par la théorie de l'enquête de John Dewey.

À Bruxelles comme ailleurs l'entrepreneuriat social ${ }^{2}$ se développe. Des organisations dédiées à l'accompagnement et à la formation d'entrepreneurs voient le jour et tissent des liens avec leur territoire. Journée

1 Christel CHRISTOPHE est doctorante en communication organisationnelle à l'UCLouvain et membre du LASCO (Laboratoire d'analyse des systèmes de communication des organisations).

2 Le présent article n'a pas pour visée de développer les différentes approches théoriques et définitions de l'entrepreneuriat social ; un concept qui s'inscrit dans un champ notionnel complexe où s'étriquent et s'enchevêtrent d'autres notions voisines telles que l'« entreprise sociale », «l'économie sociale et solidaire » (ESS), le « social business » ou encore « l'innovation sociale». À tout le moins nous suivons Mair et Martí (2006, p. 37, notre traduction) pour l'entrepreneuriat social est « un processus impliquant l'utilisation innovante et la combinaison des ressources pour saisir les opportunités qui entraînent un changement social et/ou qui répondent à des besoins sociaux $»$.

Recherches en communication, $\mathrm{n}^{\circ} 52$ - Article publié le 08/09/2021 
d'étude, soirée projection-débat, atelier... sont autant de formats disponibles pour les chargés de projet en quête de communautés entrepreneuriales. La " participation » y constitue souvent la modalité principale des échanges et contribue à rendre perceptible les préoccupations des publics. Nous avons étudié l'une de ces organisations, un centre d'accompagnement pour entrepreneurs sociaux, à un stade précoce de son développement ce qui nous a permis d'observer comment la participation y est envisagée pour permettre des dynamiques d'apprentissage. Hjorth et al. (2015) ont étudié l'émergence d'initiatives entrepreneuriales comme un processus d'apprentissage dynamique. C'est dans une perspective similaire que nous abordons ladite organisation en faisant l'hypothèse que la gestion des dispositifs participatifs a contribué à un apprentissage organisationnel des membres de l'équipe opérationnelle.

Si la notion de « dispositif participatif » a été fortement mobilisée dans les sciences politiques et les sciences de l'information et de la communication autour des enjeux de la "démocratie participative ", nous constatons que le champ de l'entrepreneuriat social adresse aussi la question de la participation des « citoyens ». À ce titre, précisons que le secteur de l'entrepreneuriat social au sein duquel opère l'organisation étudiée est marqué par des logiques expérimentales et exploratoires. Celles-ci permettent selon nous d'observer ce qui se joue en termes d'apprentissage au sein des dispositifs participatifs dont la particularité est de permettre les échanges dialogiques entre l'organisation et ses publics approchés comme des entrepreneurs ou des citoyens à écouter et sensibiliser. Nous appréhendons les événements proposés par le centre d'accompagnement comme des "dispositifs participatifs ». Eu égard de la diversité des formats proposés par le centre d'accompagnement, cet étiquetage de la réalité empirique nous permet de mettre l'accent sur les points communs constatés entre eux relatifs à la participation tout en nous permettant d'insister sur les divers agencements qui favorisent cette participation. Le terme générique de " dispositif » est familier aux SIC (Larroche, 2018) et avec lui nous désirons mettre l'accent sur une logique de médiation (Appel et al., 2010) qui suppose la communication des acteurs étudiés avec leurs publics. Couplée au concept de « dispositif», la « médiation » est entendue ici comme « ce qui permet la communication à l'autre d'idées, de savoirs, de sentiments, d'impulsions via un système commun de représentation » (Lombardo \& Angelini, 2013, p. 193). Peeters et Charlier (1999) définissent le « dispositif» comme « la concrétisation d'une intention au travers de la mise en place d'environnements aménagés » (p. 18). Notons toutefois que contraire- 
ment à Foucault qui traite du « dispositif de pouvoir » $(1975$, p. 165) ou encore de « dispositif disciplinaire » (1975, p. 205), notre approche du dispositif n'est pas centrée sur les enjeux d'une actualisation d'un pouvoir et d'une surveillance sur les acteurs mais s'intéresse à la question de la participation qui nécessite selon nous de situer la focale de l'analyse au niveau de la compréhension des dynamiques interactionnelles. Dans le champ de la communication des organisations, le « dispositif » peut servir de concept analytique à mobiliser dans « une approche processuelle de la communication qui implique un sujet/acteur et des énoncés ou des technologies, dans une relation de production de sens ou de production sociale (ou socio-organisationnelle) » (Appel \& Heller, 2010, p. 43). Il est possible d'appréhender le dispositif dans une compréhension processuelle qui permet au chercheur de rendre compte à la fois des phénomènes de «co-construction sociale/technique » et de « co-construction de sens. » (Ibid.) Enfin, si nous partageons avec Foucault un intérêt pour les dimensions techniques, procédurales et matérielles du dispositif, nous les étudierons moins comme « instrument et vecteur de pouvoir » (Foucault, 1975, p. 39) que comme instrument et vecteur de la participation, elle-même située dans les interactions.

À travers notre article, nous tentons de répondre à la question suivante : Dans quelle mesure les dispositifs participatifs permettent-ils de révéler leurs apports à une dynamique d'apprentissage organisationnelle ? Notre focale d'analyse se situe au niveau de la gestion des dispositifs participatifs par l'équipe opérationnelle en charge du centre d'accompagnement. Nous travaillons sur un matériau empirique de type ethnographique qui nous permet d'adresser le thème de la participation non pas seulement du point de vue des participants mais également de ceux qui les organisent et les mettent en œuvre (O’Miel, 2017). Un état de l'art très synthétique nous permet de pointer le lien entre la participation et l'apprentissage organisationnel par le recours à trois métaphores. Le développement empirique montre comment une intelligibilité collective se construit au sein de l'équipe opérationnelle autour d'enjeux liés à « la communauté », l'entrepreneuriat social ou encore l'organisation du travail impliqué par ces dispositifs participatifs.

\section{Trois métaphores de l'apprentissage organisationnel}

Depuis les premiers travaux sur 1'apprentissage organisationnel (March \& Simon, 1958 ; Argyris \& Schön, 1996 cités par Elkjaer, 2004 ; Cyert et March 1963 cités par Elkjaer, 2017), de nombreuses 
théories se sont développées (Chia, 2017 ; Rupcic, 2020). Celles-ci ont souvent été marquées par des conceptions dualisant « la connaissance » et « l'action ». Lorsque la focale est placée prioritairement sur la transmission de connaissance, l'apprentissage organisationnel est considéré dans sa dimension " informationnelle », c'est-à-dire en tant que résultat d'un traitement d'informations (Lorino, 2018). Cette perspective cognitiviste (Chia, 2017) conçoit l'apprentissage organisationnel à l'échelle des individus et s'intéresse à décrire comment ceux-ci acquièrent des connaissances, entre autres par la répétition d'activités ou l'accumulation de données (Lorino, 2018). Cette perspective qui s'illustre à travers la métaphore de « l'acquisition » (Elkjaer, 2004) est fondée sur une épistémologie représentationnelle et rationaliste de la connaissance. Celle-ci ne permet pas aisément de comprendre l'activité lorsqu'elle fait face à l'incertitude et n'intègre pas suffisamment dans sa compréhension de l'apprentissage les dynamiques interactionnelles. Pour répondre aux limites des conceptions rationalistes de l'apprentissage organisationnel, des approches centrées sur l'action et la pratique (practice-based learning) ont été développées à la suite de Lave et Wenger's (1991, cités par Elkjaer, 2017) et Gherardi (Rupcic, 2020). Dans cette approche, la focale y est placée sur les interactions entre les individus et l'apprentissage organisationnel est considéré à l'aulne de la participation des acteurs. Ici, le concept de participation permet de souligner le fait que l'apprentissage ne se produit pas uniquement ou principalement dans l'esprit des acteurs mais résulte davantage de leur participation à des pratiques (Gherardi, 2013) à l'œuvre dans leur organisation. Cette perspective est décrite par la métaphore de « la participation » (Elkjaer, 2017) et conçoit l'apprentissage organisationnel comme une activité sociale. Cependant, cette approche manquerait selon Elkjaer $(2004,2017)$ à expliquer comment concrètement l'apprentissage se déroule lorsque les acteurs prennent part à une pratique. Une troisième approche existe sous la métaphore de "l'enquête » (Ibid.) et vise à expliquer l'apprentissage comme un processus de résolution de situations problématiques articulant l'échelle individuelle et collective. Ce type d'approche trouve son inspiration dans la tradition pragmatiste de Ch. S. Peirce et de John Dewey et s'inscrit dans une épistémologie relationnelle et processuelle (Elkjaer, 2004, 2017 ; Martela, 2015 ; Gillberg \& Vo, 2011 ; Lorino, 2018). Ici, la participation y est vue comme ce qui permet une coordination des perspectives au sein d'un processus de (re)construction de sens (Lorino, 2013) ancré dans les pratiques et à partir duquel un travail de réflexivité est réalisé par les acteurs. Ici, les 
pratiques sont à la fois ce qui pose question et ce par quoi une construction de sens se réalise. En outre, cette approche souligne une dimension transactionnelle de l'apprentissage qui permet de saisir les relations entre les acteurs de l'organisation et son environnement comme des lieux de l'expérience où se construit une intelligibilité réciproque au moyen de la participation. Soulignons que les pragmatistes posent la participation comme une condition de l'apprentissage : « only by direct active participation in the transactions of living does anyone become familiarly acquainted with other human beings and with « things » that make up the world » (Dewey, 1949). Nous situons notre travail dans cette dernière approche et proposons d'interpréter les dispositifs participatifs comme ce qui (1) favorise une logique transactionnelle entre les acteurs de l'organisation étudiée et ses publics et (2) permet de générer une construction de sens dans et sur les expériences vécues en lien avec la pratique de gestion des dispositifs participatifs.

\subsection{Cas d'étude, données et méthodes}

Juillet 2014, une fédération belge d'économie sociale introduit avec six organisations partenaires une candidature dans le cadre de la programmation FEDER 2014-2020. Le projet décrit dans le document de candidature consiste en la mise en place d'un centre « centre d'entrepreneuriat social, coopératif et collaboratif » à Bruxelles. Au sein du centre, des collectifs d'entrepreneurs sociaux peuvent, après une procédure de sélection, bénéficier d'un soutien à la création de leur projet entrepreneurial. L'offre d'accompagnement est divisée en quatre programmes, chacun adapté au stade d'avancement et à l'ampleur des projets entrepreneuriaux : Start, Grow up, Challenge, Mutalize ${ }^{3}$. L'équipe opérationnelle en charge du suivi quotidien des activités du centre est composée d'employées qui ne se connaissaient pas avant de rejoindre le projet et ont été embauchées par les différentes organisations partenaires. À l'automne 2016, l'équipe opérationnelle se complète et le projet prend une forme plus concrète avec le lancement de la première sélection de projets entrepreneuriaux. À cette même période, les membres de l'équipe emménagent dans des locaux nouvellement rénovés et se préparent à ouvrir l'espace de coworking mis à disposition des entrepreneurs. Entre 2016 et 2018, l'équipe ${ }^{4}$ est composée d'une

3 Nous proposons ici des noms d'emprunt pour identifier les programmes.

4 Nous proposons ici des noms d'emprunt pour identifier les acteurs. 
coordinatrice (Valérie), trois responsables de programme (Hélène, Sophie et Diane), une responsable du coworking (Amélie) et une assistante administrative (Josiane). En partir de 2018, des changements ont lieu dans la composition de l'équipe et la fonction de responsable du coworking a été revue pour répartir le travail en deux fonctions : une responsable animation et communauté (Charlie) et une responsable du coworking (Anaïs). Dès l'ouverture du centre, des activités mobilisant une approche participative sont proposées aux entrepreneurs accompagnés et à des publics externes. Nous avons pris part à certaines des activités qui font l'objet de cet article et nous avons observé les réunions hebdomadaires de l'équipe ayant ainsi accès aux coulisses de la pratique. Nos analyses reposent sur les données ethnographiques issues de cette étude de cas parmi lesquelles une sélection a été réalisée afin d'y extraire celles qui se rapportent à la pratique étudiée. Nous avons organisé les données chronologiquement de manière à (1) faire l'inventaire des événements organisés à partir des informations contenues dans les newsletters et sur la page Facebook de l'organisation, (2) catégoriser les types d'événements en fonction du contenu des annonces (« soiréedébat », « soirée pitch », " atelier ») et (3) associer à l'inventaire des événements les autres données s'y rapportant telles que celles issues de l'observation, d'interviews et de documents. Le tableau ci-dessous reprend une vue simplifiée de l'inventaire des événements organisés entre 2016 et 2019.

Tableau 1. Inventaire des événements organisés par le centre d'accompagnement pour la période 20.06.2016-01.03.2019

\begin{tabular}{|c|l|c|}
\hline $\begin{array}{c}\# \\
\text { Événements }\end{array}$ & \multicolumn{1}{|c|}{ Type } & $\begin{array}{c}\# \\
\text { Observations }\end{array}$ \\
\hline $\mathbf{9}$ & Atelier/formation & $\mathbf{3}$ \\
\hline $\mathbf{6}$ & $\begin{array}{l}\text { Cérémonie/Événement de lancement ou clôture de } \\
\text { programme }\end{array}$ & $\mathbf{4}$ \\
\hline $\mathbf{1 4}$ & Rencontre et témoignage & $\mathbf{5}$ \\
\hline $\mathbf{1 4}$ & Séances d'information sur les accompagnements & $\mathbf{2}$ \\
\hline $\mathbf{3}$ & Séminaire & $\mathbf{0}$ \\
\hline $\mathbf{1 1}$ & Soirée pitch et rencontre & $\mathbf{3}$ \\
\hline $\mathbf{5}$ & Débat & $\mathbf{1}$ \\
\hline
\end{tabular}

Différents types de données ont été exploitées afin de rendre compte du phénomène d'apprentissage. Correspondant à des espaces 
de conversation spécifiques et permettant d'articuler différentes orientations temporelles, les données sont issues des documents suivants : notes d'observation, documents, entretiens de recherche. Cette articulation permet selon nous une analyse d'un point vue transactionnel et processuel. Le tableau ci-dessous reprend l'inventaire des types de données exploitées dans le cadre de la présente réflexion.

Tableau 2. Inventaire des types de données pour la période 20.06.201601.03 .2019

\begin{tabular}{|c|c|}
\hline Type de données & Format de données \\
\hline Données issues de documents publics & $\begin{array}{l}\text { Documents promotionnels } \\
\text { Captures d'écrans du site internet } \\
\text { Annonces d'événements sur Facebook } \\
\text { Documents diffusés lors des événements }\end{array}$ \\
\hline Données issues de documents internes & $\begin{array}{l}\text { Notes internes } \\
\text { Documents stratégiques }\end{array}$ \\
\hline Données issues de l'observation & $\begin{array}{l}\text { Notes d'observation } \\
\text { Photographies } \\
\text { Verbatim }\end{array}$ \\
\hline Données issues d'interviews & Retranscriptions d'entretiens \\
\hline
\end{tabular}

\section{Restituer des interprétations}

\subsection{Expérimenter la relation avec le public}

La programmation d'événements, initialement réalisée au gré des opportunités, ne semblait pas répondre à des objectifs stratégiques fixés ex ante. L'engagement d'une responsable de coworking, formée en facilitation et animation de groupe, a favorisé l'opérationnalisation de l'objectif « création d'une communauté entrepreneuriale ${ }^{5}$ » grâce

5 Cet objectif est brièvement évoqué dans le dossier de candidature du projet : « mettre en place un écosystème favorable à la création d'entreprises et d'emplois dans l'entrepreneuriat social, coopératif et collaboratif, en permettant aux entrepreneurs et porteurs de projet, d'avoir un accès facilité à un accompagnement spécifique, à l'expertise, aux bonnes pratiques, au financement et à des réseaux, et ce au travers d'une offre intégrée d'accompagnement et de formation destinée aux entrepreneurs et à l'organisation en réseau d'une communauté d'entrepreneurs sociaux et d'économie collaborative » (Extrait du dossier de candidature). 
1 [AMÉLIE] : Mais donc la deuxième partie en effet de mon boulot c'était

2 euh... c'était de développer une communauté. [...] Et alors, ce qui

3 est intéressant c'est que, quand même, je sais pas si je t'avais raconté l'histoire

4 du, du poste ? C'est qu'au début c'était un poste d'accueil, gestion du lieu et

5 accueil. Donc, en gros, assistante d'accueil quoi, donc : « Bonjour, bienvenue

6 chez [Organisation] : vous voulez voir quel entrepreneur ? Voilà du café.

7 Voilà une salle d'attente ». Et en fait, ça c'était quand ils [les partenaires du

8 projet] cherchaient un lieu et tout ça, et puis ils sont arrivés chez [Organisation

9 locatrice], et [Organisaton locatrice] a dit: Bah ok, mais nous on peut vous

10 faire l'accueil. » Donc pas besoin. Donc ils [les partenaires du projet] ont dit :

11 «Ah ben ok, avec l'argent qu'il reste et le temps que cette personne aura, on va

12 dire qu'elle n'a qu'à s'occuper de la communauté ». Donc, c'était un boulot, une

13 partie de métier qui était pas du tout un besoin prioritaire de [Organisation], ils

14 n'avaient à aucun moment, dans l'appel qui a été écrit pour le FEDER, il n'y a

15 jamais eu de réflexions sur la communauté, c'était de l'acompagnement

16 d'entrepreneurs, coopératif, à travers des, des modules d'accompagnement

17 tout ça sans réfléchir au lien entre les gens. [...] Et donc, oui, la question de

18 la communauté c'était en fait, c'est, ça veut dire quoi pour nous faire

19 communauté ? Parce qu'en fait, ce qui était intéressant c'est que vu que ce

20 boulot avait été mis en place comme ça, hop, fais ça, j'avais aucun cadre. Il n’y

21 avait pas de stratégie, il y avait, ça venait de nulle part quoi. Moi je demandais

22 à [VALÉRIE]: « Mais c'est quoi communauté, vous voulez que je fasse quoi ? »

23 «Ah non mais justement il faut réfléchir. »

\section{Extrait 1. Entretien avec la responsable du coworking}

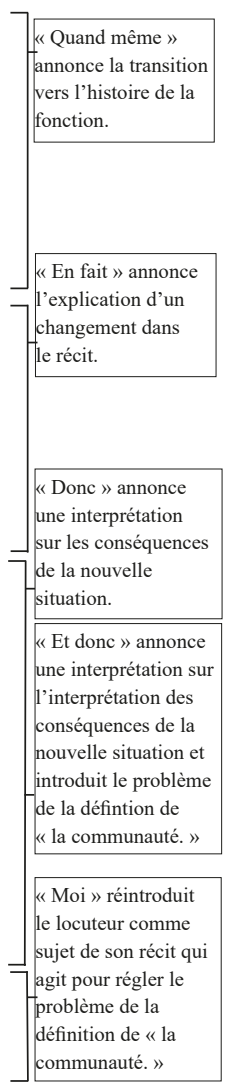

à une approche centrée sur des animations participatives. La première newsletter de l'organisation en juillet 2016 mentionne en filigrane des « animations » considérées comme un élément constitutif d'un « environnement propice » à la création d'entreprise sociale. Mais, comme l'illustre l'extrait d'entretien, les incertitudes quant aux manières de « faire communauté ${ }^{6}$ » ont marqué la pratique de gestion de dispositifs

6 Nous avons pu observer que l'expression " communauté » était utilisée par les acteurs sur le terrain. Appartenant à un discours indigène, cette notion ne faisait pas pour autant consensus sur la manière de l'appréhender. Parallèlement, cette expression existe aussi comme un concept scientifique dont la portée analytique varie considérablement en fonction des ancrages théoriques à partir desquels il est mobilisé. Dans le cadre de cet article, nous n'avons pas pour objectif de faire l'état de l'art autour de cette notion fortement polysémique, préférant faire émerger le sens que ce terme a pour les acteurs. Notre approche inspirée du pragmatisme nous invite à en 
participatifs ouvrant ainsi la voie à une attitude expérimentale pour les membres de l'équipe.

L'extrait ci-dessus propose un regard rétrospectif sur la prise de fonction de la responsable du coworking et revient sur la genèse des missions confiées. Il permet de comprendre la nature de l'incertitude quant à la gestion de dispositifs participatifs. Celle-ci se rapporte à la définition du concept de « communauté » ainsi qu'aux méthodes de travail à mettre en place pour atteindre un des objectifs de la fonction. Plusieurs niveaux d'analyse sont possibles. Les marqueurs discursifs signifiés par les expressions « quand même », « et en fait », 《 donc », « et donc » et « moi » permettent de faire ressortir la structure macrosyntaxique de l'extrait et de lire l'organisation des idées comme suit : (1) il est utile de parler de l'histoire de la fonction (2) pour comprendre que la gestion de la communauté n'était pas une mission anticipée (3) ce qui rend compliqué la prise de fonction (4) et nécessite une démarche de clarification.

Cette donnée évoque les publics de l'organisation tels que figurés (Roginsky \& Christophe, 2018) sous la notion de « communauté »; notion qui semble être ambigüe pour les acteurs. Comme le notent Roginsky et Christophe (2018, p. 138) : « Face à l'évanescence des publics, il est nécessaire pour les membres de l'organisation de recourir à la figuration pour engager leur point de vue dans la discussion et faire parler leur expérience des publics. » Dans ce cas-ci, la situation initiale liée à la pratique de gestion de dispositifs participatifs appelle un besoin de compréhension mutuelle sur la notion de communauté qui, nous le verrons, nécessite pour les acteurs d'être en contact avec les publics de l'organisation. Pour le chercheur, il est intéressant d'observer comment les dispositifs participatifs sont saisis comme une modalité de l'enquête. À partir de l'extrait suivant, nous étudions comment les séances d'information mises en place dans une phase de prospection de projets entrepreneuriaux à accompagner ont été vécues comme une sorte de « validation » de la démarche du centre.

considérer cette expression comme un « signe » dont la signification reflète des habitudes d'action qu'il convient pour le chercheur de mettre au jour. Nous appréhendons donc davantage la notion à partir de son contexte pragmatique en essayant de comprendre comment elle est située dans l'action des acteurs. Soulignons toutefois que Ch. S Peirce et John Dewey ont élaboré des réflexions autour de cette notion, notamment à partir du concept de « communauté d'enquête ». 
1 Ouais... j'avais mis, ouais moi j'ai mis la première séance d'info parce qu'à la fois c'était

2 vraiment une soirée particulière mais plus que ça, c'était vraiment de dire « est-ce qu'on

3 va trouver notre public ? », finalement, « est-ce qu'on va valider qu'il y ait une vraie

4 demande » ? Est-ce que, est-ce que ce qu'on propose intéresse les gens ? Ouais, on avait

5 fait pas mal de rencontres avec les, les porteurs de projet et c'était un peu un quitte ou

6 double : est-ce que les gens avaient vraiment envie de nous rejoindre pour 8 mois quoi ?

7 Voilà... C'était une façon de rencontrer qu'est-ce qu'il y a comme projets en émergence à

8 Bruxelles. Voilà, outre la soirée qui était un moment particulier.

Extrait 2. Entretien collectif avec les membres de l'équipe opérationnelle

Cet extrait d'entretien mené en juillet 2017 reprend la parole de la coordinatrice du projet. La conversation provoquée et encouragée par la situation d'entretien révèle une forte dimension réflexive. À ce titre, elle peut être considérée comme une méta-conversation (Robichaud et al., 2004). Nous n'avons pas participé aux discussions préparatoires du projet mais les quatre questions posées par la coordinatrice prennent la forme d'un discours rapporté que nous supposons être antérieur au moment de l'énonciation. «Est-ce qu'on va trouver notre public ?» (1. 2-3), «Est-ce qu'on va valider qu'il y ait une vraie demande ? » (1. 3-4), « Est-ce que ce qu'on propose intéresse les gens ? » (1. 4-5) et « Est-ce que les gens avaient vraiment envie de nous rejoindre ? (1.6) sont des questions qui ventriloquent (Cooren, 2010 ) « on » et « nous », soit un acteur collectif que nous supposons être le collectif en charge de la création du projet de création du centre d'accompagnement. Ces questions mettent en exergue les préoccupations de la coordinatrice et, ce faisant, précisent les incertitudes qui caractérisent le démarrage du projet. L'isotopie syntaxique qui consiste pour le locuteur à répliquer quatre fois la même formulation « est-ce que ? » rend encore plus évident le procédé de validation des hypothèses de travail qui peuvent se résumer


tés, alors (1) « on » va trouver « notre » public, (2) « valider » la présence d'une demande, (3) intéresser le public et (4) l'enrôler dans le projet.

Le nombre de participants aux événements a été un indicateur important pour les membres de l'équipe dans le cadre des réflexions sur la pertinence de l'offre d'un programme d'animation. Nous avons noté que l'importance de la valeur chiffrée s'est traduite en cours de projet dans différentes méthodes de collecte de données, notamment par le recours à des listes de présence lors des événements. Mais, avant de devenir une «donnée » de quelque forme que cela soit, la présence des participants peut aussi être comprise comme un élément constitu- 
tif d'une expérience de rencontre avec les publics de l'organisation. L'extrait suivant illustre la dimension qualitative de la relation entre les membres de l'équipe et les publics. Le «Pitch and Meet » dont il est question se rapporte à un événement que nous catégorisons comme " soirée pitch et rencontre 》 et qui met généralement en présence des entrepreneurs en cours de projet avec des entrepreneurs à un stade précoce de la réflexion. Ce type de rencontre est soutenu par des dispositifs participatifs déclinant la prise de parole en grand groupe et en petits groupes tout en favorisant les déplacements libres dans l'espace.

1 Le «Pich and Meet» avec [organisation partenaire], le deuxième où j'ai vraiment

2 participé toute la soirée. Et ça été génial pour moi de voir toute cette communauté

3 qui avait grossi en un coup. Voilà, tous ces gens qui venaient à [Organisation] c'était

4 un peu... Voilà, c'est une donnée. En fait, quand on est entrepreneur, on est

5 entrepreneur dans l'entrepreunariat social et qu'on veut rencontrer d'autres

6 entrepreneurs dans le même domaine, on vient à [Organisation]. C'est comme ça.

7 Et il y avait plein de liens qui se faisaient dans tous les sens et ça je trouvais

8 incroyable. Voilà. I think. I think, c'étaient mes moments marquants. Bon, j'ai pas

9 parlé de l'inauguration parce que j'allais pas faire comme tout le monde.

\section{Extrait 3. Entretien collectif avec les membres de l'équipe opérationnelle}

La personne qui s'exprime dans l'extrait est co-gestionnaire du programme [Start]. L'extrait permet d'analyser comment l'énoncé du locuteur construit des jugements conclusifs ancrés dans l'expérience de la relation au public. L'interprétation de ces différentes conclusions est appuyée par une analyse de la séquence interprétative qui peut être détaillée comme suit :

\section{Jugement 1}

\begin{tabular}{|c|c|c|c|}
\hline $\begin{array}{l}\text { «J'ai vraiment } \\
\text { participé » (1. 1) }\end{array}$ & $\begin{array}{l}\text { «Et ça été génial } \\
\text { pour moi » }(1.2)\end{array}$ & $\begin{array}{l}\text { «Voilà tous ces } \\
\text { gens" }(1.3)\end{array}$ & $\begin{array}{l}\text { "Voilà, c'est une } \\
\text { donnée » (1. 4) }\end{array}$ \\
\hline $\begin{array}{l}\text { Indique des garanties } \\
\text { de véracité : le } \\
\text { propos se base sur } \\
\text { une expérience } \\
\text { authentique. }\end{array}$ & $\begin{array}{l}\text { Indique l'expres- } \\
\text { sion d'une émotion } \\
\text { associée à la surprise } \\
\text { et à l'appréciation de } \\
\text { l'expérience. }\end{array}$ & $\begin{array}{l}\text { Explicite ce qui était } \\
\text { «génial » en se basant } \\
\text { sur le constat d'une } \\
\text { présence en nombre } \\
\text { de participants. }\end{array}$ & $\begin{array}{l}\text { Interprète le constat } \\
\text { comme une donnée } \\
\text { de l'expérience. }\end{array}$ \\
\hline
\end{tabular}

Participer aux événements permet de générer des données 


\section{Jugement 2}

(5)

« En fait, quand on est entrepreneur » $(1.4$ à 6$)$

Explique (« en fait») ce à quoi se rapporte la «donnée » évoquée dans la séquence précédente par la mise en scène d'un entrepreneur généralisé sous le le pronom « on »..
(6)

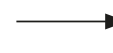

« On vient à [Organisation] » (1. 6) d'autres entrepreneurs » (1. 5 à 6)

Généralise la valeur perçue de ce type d'événement pour un entrepreneur type, c'està-dire de pouvoir « rencontrer d'autres entrepreneurs ».
Interprète le rôle de l'organisation par rapport aux attentes d'un entrepreneur type.

[Organisation] répond au besoin de socialisation des entrepreneurs

\section{Jugement 3}

(8)

« Et il y avait plein de $\underline{\text { liens } ~ » ~}$ (1. 7)

Indique comment la séquence précédente est interprétée. La valeur de la rencontre est identifiée comme une manière de «faire des liens », de développer des idées.
(9)

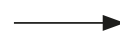

(10)

« Je trouvais ça incroyable » «Bon » (1. 9) (1. 7 à 8$)$

Indique l'expression d'une Marque une clôture du proémotion associée à l'ap- pos et une ouverture sur un préciation de l'expérience. autre propos. Interprète la valeur du constat précédemment indiqué comme positif.

Le rôle de [Organisation] dans la mise en lien entre les entrepreneurs est positivement valorisé

L'extrait indique que la soirée «Pitch and Meet » était marquante de par la rupture qu'elle a introduit dans l'expérience de la relation au public : un étonnement, une surprise qui a permis de focaliser une attention sur la valeur des événements. La rupture s'exprime dans le registre de l'abondance et du mouvement : « toute cette communauté qui avait grossi d'un coup » (1.2 à 3); " Tous ces gens qui venaient » (1.3); «Il y avait plein de liens qui se faisaient dans tous les sens » (1. 7). Les descriptions du locuteur semblent permettre aux allocutaires (ici les membres de l'équipe participant à l'entretien collectif) de revivre le moment. Cette description permet d'illustrer le potentiel de médiation du dispositif (Appel et al., 2010) qui dans ce cas-ci permet à la fois de 
créer des liens entre les différents sujets portés par les « gens » présents à l'événement tout en donnant une impression de décentralisation des échanges qui « se faisaient dans tous les sens ». Cette description joue également sur le sens visuel grâce à différents procédés dont la métonymie et la métaphore : les participants sont associés à la "communauté » qui est elle-même associée à une entité capable de changer de volume ; la métaphore du « lien » qui dans l'extrait peut se rapporter aux interactions entre les participants.

Cette figuration (Cooren, 2010) des participants aux événements permet de les ventriloquer sous différents termes oscillant du plus abstrait au plus concret : « la communauté » (1. 2), « ces gens » (1. 3), « on » (1. 4), « les entrepreneurs » (1.6). La nature métonymique de la description où les participants sont appréhendés comme la partie d'un tout (« la communauté », « les entrepreneurs ») qui existe non pas à la portée expérientielle mais dans le discours relève de la figuration. Le procédé permet d'enrichir le sens donné aux objets de l'interprétation tout en exploitant l'expérience vécue comme matrice du discours. Le procédé de figuration permet d'abstraire de l'expérience un jugement évaluatif sur l'action passée et ce faisant, la figuration du public induit des orientations pour l'action future ou, dit autrement, montre son agentivité (Jolivet \& Vasquez, 2011) qui participe selon nous à l'apprentissage organisationnel. À ce titre signalons que l'activité d'enquête est une « combinaison étroitement intégrée d'action et d'interprétation de l'action » (Lorino, 2007, p. 768).

\subsection{Rendre visibles des traces de la participation}

Pour les membres de l'équipe, apprendre à connaître les publics des événements proposés va de pair avec apprendre à élaborer des formats de rencontre qui répondent à leurs préoccupations. Médiatisée par le dispositif participatif, la relation entre les membres de l'équipe et les publics des événements est également porteuse d'apprentissage sur la manière dont ceux-ci appréhendent les thèmes de discussion proposés. En effet, le contact avec les participants aux événements offre les conditions d'une mise à l'épreuve de la notion d' « entrepreneuriat social » qui, comme nous l'ont renseigné les membres de l'équipe, a été difficile à comprendre et à faire comprendre. À l'instar de l'exemple présenté ci-dessous, les événements de type « atelier / formation » étaient particulièrement adaptés pour des discussions de fond sur la terminologie et les enjeux de l'entrepreneuriat social. 


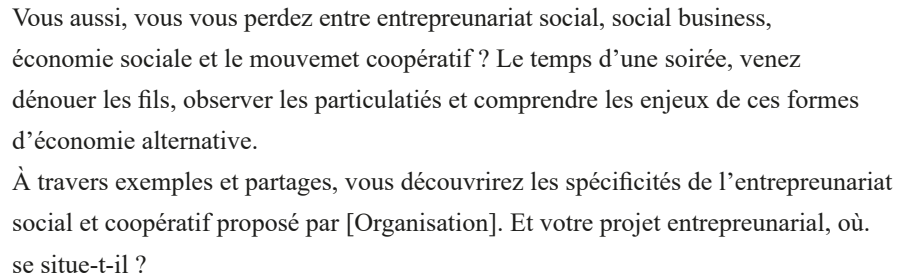

Extrait 4. Annonce d'un événement. B.A.-BA de l'entrepreunariat social

Dans le cas de l'atelier «B.A - BA de l'entrepreneuriat social » les ambiguïtés terminologiques et les défis de compréhension sur l'« entrepreneuriat social » participent à la mise en place du dispositif participatif. L'hétérogénéité des perspectives y est saisie comme une ressource dans le cadre des échanges avec des participants aux profils variés : entrepreneurs (sociaux) confirmés ou débutants, professionnels du secteur associatif ou privé, travailleurs en quête de reconversion, curieux, étudiants, chercheurs... Nos observations ont révélé que l'exploration de cette notion par les membres de l'équipe intégrait les apports de la participation des publics auxquels s'adressent in fine les efforts de définition.

Cette posture ouverte aux questionnements et qui sollicite la contribution des participants dans le contenu même des animations a souvent été constatée. Les documents internes utilisés pour l'organisation et la conduite des dispositifs participatifs reprenaient fréquemment des informations telles que le découpage de l'animation en tranches horaires, les thèmes de discussion, les modalités de répartition de la parole et les questions utilisées comme inducteur de la participation et de la mise en commun des réflexions. Nous avons noté le rôle structurant des questions soumises aux participants oralement ou affichées sur de grands post-it. Celles-ci permettaient aux animateurs d'organiser les échanges tout en ouvrant un espace pour faire parler les participants : «Qui êtes-vous? », «Quels est votre projet? », « Quelles questions pensez-vous avoir sur l'entrepreneuriat social? ». Les participants sont alors invités à agir et à partager leur point de vue de différentes manières : se positionner dans l'espace pour signifier une proximité ou une distance par rapport à un sujet, déambuler parmi les participants et engager la conversation sur un thème, noter sur des post-it ou sur un tableau des mots clés, discuter en petits groupes de projets entrepreneuriaux, se désigner comme porte-parole et restituer l'état d'une réflexion menée avec les autres participants... Les flux de conversations don- 
naient l'impression d'osciller dans un mouvement qui centralisent et décentralisent des informations collectées, partagées, verbalisées, dessinées, écrites et souvent affichées sur les murs.
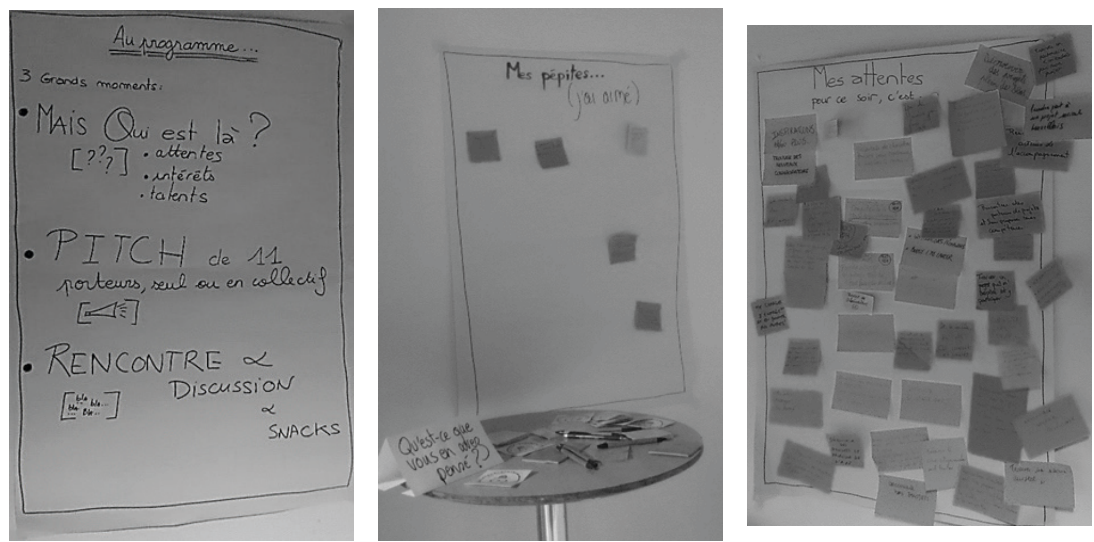

Illustration 1. Affiches disposées lors des événements

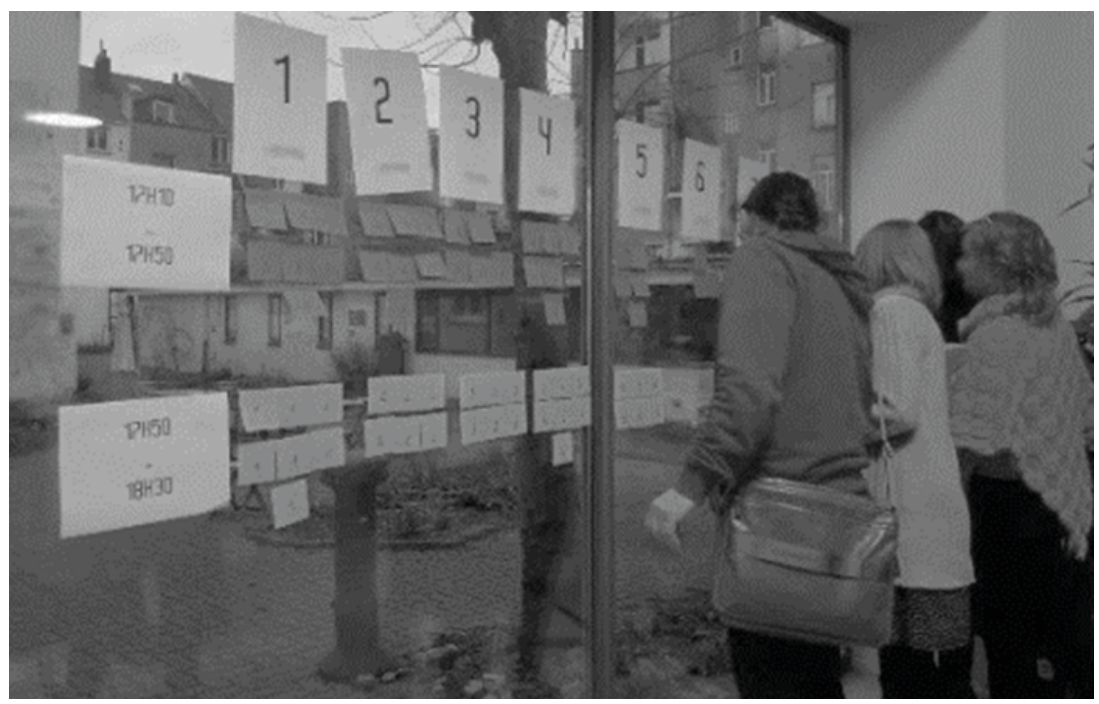

Illustration 2. Des participants sont invités à sélectionner des sujets de discussion

Équipés pour extérioriser et faire parler les représentations des participants, les dispositifs mis en place invitent à la participation grâce aux « arrangements spatiaux et sémiotiques » (Meunier, 1999) qui suggèrent différentes modalités de médiation de la relation des acteurs 
en présence. Ce faisant, ils définissent des espaces de dialogue et permettent aux membres de l'équipe d'observer ce qui se dit au sein des interactions.

La ligne 5 de la capture d'écran montrée en début de section précise : « à travers des exemples et partages, vous découvrirez les spécificités de l'entrepreneuriat social ». Ceci indique une autre modalité communicationnelle observée lors des animations qui est l'exemplification de pratiques entrepreneuriales à partir des témoignages des participants. Ce qui est alors « découvert» l'est autant pour les participants que pour les membres de l'équipe présents aux animations. Dans le même ordre d'idée, les exemples de projets entrepreneuriaux qui s'incarnent dans les pitches permettent aux membres de l'équipe de construire à la fois des connaissances sur la « communauté d'entrepreneurs » et sur l'objet «l'entrepreneuriat social».

Les dispositifs mis en place visent à structurer les relations de communications entre les acteurs (Meunier, 1999) et à cadrer l'organisation de l'action (Peeters et Charlier, 1999) en vue de l'accomplissement de certaines fins anticipées par les organisateurs. Ainsi, alors que chacun vient dans les événements avec son propre intérêt, ses motivations personnelles, nous avons pu observer des efforts de mise en commun grâce aux modalités communicationnelles que nous avons indiquées (questionnement, pitch, synthèse de discussion, traces écrites) et qui reposent sur la participation des publics. La mise en commun des expériences, la mutualisation des connaissances, la confrontation des idées laissent ensuite des traces que chacun peut saisir pour son propre projet.

\section{Mettre en commun les données de l'expérience par la participation}

Comme le précise Larroche (2018) les dispositifs sont reproductibles et cette reproductibilité a permis selon nous aux membres de l'équipe opérationnelle de développer des habitudes et de trouver des réponses aux questions que ces mêmes dispositifs ont initialement permis de générer. Bien que ce que le dispositif participatif produit est à chaque fois unique, étant donné que ce qui se dit, ce qui se fait lors des événements dépend d'une configuration à un moment donné, les traces engendrées dans et par la participation peuvent quant à elles être mobilisées dans les réflexions de l'équipe opérationnelle. En termes d'apprentissage organisationnel, ces dispositifs participatifs créent des traces d'expériences collectives mobilisables dans une logique d'enquête. Ils permettent de rendre visibles les publics, les questionnements 
récurrents, les ressources attendues ou disponibles ou encore les projets entrepreneuriaux en cours de réflexion. Jeanneret (2013), inspiré entre autres par Ch. S. Peirce explique que l'effet trace est « un construit communicationnel » qui est produit au cours « d'une genèse sociale et interprétative qu'il s'agit de mettre au jour » (Ibid., p. 42) La dynamique d'enquête dont nous essayons de rendre compte ici s'apparente à ce type de processus d'interprétation. La trace peut y être mobilisée pour engager « une lecture des comportements et des objets orientés vers leur passé » (Ibid., p. 47). Toutefois, aucune trace n'existe indépendamment d'une « stratégie interprétative (herméneutique) particulière qui doit être socialement partagée pour être opérante » (Jeanneret, Ibid.). Dit autrement, pour qu'une trace devienne un signe qui indique quelque information par rapport à l'objet auquel il se rapporte, il faut qu'il soit interprété comme tel, c'est-à-dire qu'il soit mobilisé dans un processus d'inférence. Cette conception de la trace n'est pas loin d'une lecture pragmatiste de l'expérience qui serait à la fois « matériel » et « méthode » pour l'enquête. C'est dans l'expérience que sont puisés les éléments sur lesquels faire des inférences et c'est l'expérience qui indique ce qui pose problème et nécessite de faire enquête. Les traces constituées grâce à la participation des publics peuvent devenir des données qui guident les acteurs dans leur compréhension des enjeux de la programmation d'événements en vue de la création d'une communauté d'entrepreneurs. Comme le précise Dewey " être une donnée, c'est avoir une fonction spéciale dans le contrôle de l'objet de l'enquête » (1993 (1938), p. 192). À travers un extrait issu de nos notes d'observation, nous explorons la manière dont les membres de l'équipe discutent de leur pratique de gestion de dispositifs participatifs lors d'une réunion hebdomadaire. Cet extrait montre comment l'enquête autour de la « communauté » peut se traduire dans une expérience collective à l'échelle de l'équipe opérationnelle.

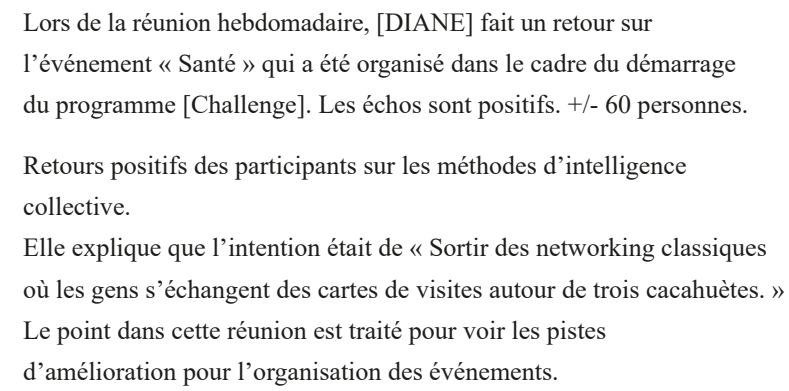


10 L'animation lors de l'événement a dû être prise en charge par [DIANE]

11 et [VALÉRIE] car [AMÉLIE] était malade : « C'est bien qu'on l'ait

12 fait finalement, car on sait le faire $»$.

13 Dans le cadre de cette discussion sur le retour d'événement, on y parle

14 des « pitches » sur [Organisation] : « Il faudrait prévoir des pitches

15 différents en fonction du public. »

16 D'autres pistes d'amélioration sont discutées : création d'une

17 procédure pour l'archivage des listes de présences aux événements

18 pour les indicateurs de projet FEDER.

Extrait 5. Notes d'observation 08.03.2017. Réunion d'équipe

L'extrait ci-dessus montre comment le retour en équipe sur un événement est mobilisé en tant qu'une ressource dans le cadre d'une démarche d'amélioration continue. Aux lignes 10 à 12, nous pouvons lire que l'animation n'a pas été prise en charge par Amélie, la responsable du coworking. Ce changement de dernière minute a permis aux deux autres collègues de se confronter aux techniques d'animation de groupe : "C'est bien qu'on l'ait fait finalement, car on sait le faire. » (1. 11 à 12). Pour l'apprentissage organisationnel, cette situation crée un précédent qui justifie une compétence mobilisable dans le futur. La proximité des verbes « faire » et «savoir » dans le verbatim pris à la volée appuie l'argument d'une connaissance émergente de l'action. Cette situation initialement vécue comme inconfortable a été discutée en équipe et, suite à cela, un principe de « back-up » a été mis en place, comme l'atteste le PV de la réunion : " Avoir un rôle très clair de chaque event = avoir un principe de backup au cas où il y ait des absents. » Cet événement n'est pas anodin. En effet, la compétence d'animation de groupe est fortement stratégique pour l'organisation et le fait de se former ou de s'initier à ces méthodes est positivement évalué par les membres de l'équipe. D'autant plus qu'il y a des « retours positifs des participants sur les méthodes d'intelligence collective » (l. 4 et 5). À nouveau, le retour des participants devient une donnée pour la réflexion collective et participe à la production de sens autour de ces dispositifs et, plus largement, sur la posture de l'organisation vis-à-vis de ses publics. À ce titre, Gillberg et Vo (2011) notent que « nous créons de la connaissance si les diverses expériences de nos vécus nous permettent de donner un sens à certains résultats concrets de nos actions » (p. 417, notre traduction).

Un autre point abordé lors de la réflexion (1. 13 à 15) concerne la question du matériel de communication à produire dans le cadre des 
événements et la clarification sur les manières de présenter l'organisation. Ce point est introduit sous la forme de la proposition : « Il faudrait prévoir des pitches différents en fonction des publics. » Il est utile de rappeler ici que la pratique du pitching est fortement associée au travail de prospection et de promotion des entrepreneurs. Cette pratique consiste à énoncer un petit discours formaté, clair et concis sur l'identité de l'entrepreneur ou sur le produit. Dans le cadre des animations, les membres de l'équipe ont fréquemment sollicité les entrepreneurs à faire le « pitch» de leur projet. Cela peut expliquer le besoin des membres de l'équipe de développer également un " pitch » sur l'organisation. En l'occurrence, les pitches sont envisagés dans une approche qui permet de les décliner en fonction des publics. Cet élément semble indiquer qu'une connaissance des publics se construit et, en termes d'apprentissage organisationnel, cette réflexion illustre selon nous les effets de la caractéristique transactionnelle des dispositifs participatifs.

\section{Conclusion}

À travers notre contribution, nous avons tenté de montrer comment la participation des publics au sein d'événements organisés par un centre d'accompagnement pour entrepreneurs sociaux permet d'engendrer des apprentissages collectifs à l'échelle d'une équipe opérationnelle. Si dans le cadre de cet article nous n'avons pas eu l'occasion d'illustrer une production matérielle concrète qui découle de ces apprentissages, nous avons tenté de montrer comment ceux-ci se produisent au croisement des expériences entre les acteurs qui, en échangeant sur leur vécu dans le cadre d'évènements configurés par des dispositifs censés faciliter la participation, créent les conditions d'une intelligibilité réciproque. En outre, nous avons tenté de montrer la manière dont ces dispositifs contribuent à rendre visibles les traces d'expériences collectives mobilisées par la suite dans le cadre des réflexions des membres de l'équipe. Lorino rappelle, en pointant les limites des approches rationalistes, que « les thèmes de la connaissance et de l'apprentissage collectif parcourent les sciences des organisations depuis des décennies, tant ils s'avèrent incontournables pour quiconque, chercheur ou praticien, cherche à comprendre les performances contrastées des organisations et leurs dynamiques d'évolution » (2007, p. 754). Notre contribution nous a permis d'esquisser une approche info-communicationnelle d'inspiration pragmatiste intégrant la participation comme vecteur d'apprentissage. Pour des futures recherches en communication 
des organisations qui visent à intégrer l'étude des dispositifs dans le cadre de phénomène organisationnel (dont l'apprentissage fait partie), l'apport d'une démarche d'analyse centrée sur les activités d'enquête insiste sur le fait qu'apprendre est un processus vivant. Un processus qui se développe dans la réciprocité entre le « faire » et le « penser» et qui toujours est situé d'une manière ou d'une autre dans le concret des situations vécues. Au sein de ce processus, la communication joue un rôle fondamental car elle est ce qui permet, à partir des expériences, d'articuler les multiples perspectives en présence. Dewey considère un effort de communication comme aboutit lorsqu'il permet aux parties en présence d'intégrer d'une manière réciproque les perspectives sur les objets convoqués par le discours. La communication réussie ne nécessite pas d'imposer un point de vue ou de faire sien le point de vue de l'autre mais plutôt de pouvoir l'intégrer comme une donnée permettant la coordination dans l'action. Notons encore que Dewey envisagea la communication comme le processus de création de la participation :

For communication is not announcing things, even if they are said with the emphasis of great sonority. Communication is the process of creating participation, of making common what had been isolated and singular; and part of the miracle it achieves is that, in being communicated, the conveyance of meaning gives body and definiteness to the experience of the one who utters as well as to that of those who listen. (Dewey, 1934, 248)

Cette manière d'appréhender la communication se prolonge dans une conception deweyenne de la «communauté » où ce qui est commun est « le fruit de la contribution individuelle de toutes les personnes concernées, et non la condition préalable de leur coordination » (Zask, 2008, p. 183).

\section{Bibliographie}

Appel, V., Boulanger, H. \& Massou, L. (2010). Dispositif [s] : discerner, discuter, distribuer. Dans V. Appel, H. Boulanger \& L. Massou (dir.), Les dispositifs d'information et de communication. Concepts, usages et objets. Coll. Culture \& Communication (pp. 9-16). Bruxelles, De Boeck.

Appel, V. \& Heller, T. (2010). Dispositif et recherche en communication des organisations. Dans V. Appel, H. Boulanger \& L. Massou (dir.), Les dispositifs d'informa- 
tion et de communication. Concepts, usages et objets. Coll. Culture \& Communication (pp. 39-57). Bruxelles, De Boeck.

Chia, R. (2017). A Process-philosophical Understanding of Organizational Learning as "Wayfinding": Process, Practices and Sensitivity to Environmental Affordances. The Learning Organization, 24(2), 107-118. Doi : https://doi.org/10.1108/TLO-112016-0083

Cooren, F. (2010). Ventriloquie, performativité et communication. Ou comment fait-on parler les choses. Réseaux, 163(5), 33-54. Doi : https://doi.org/10.3917/res.163.0033

Dewey, J. (1993 [1938]). Logique de l'enquête. Paris : PUF.

Dewey, J., Boydston, J.A. (eds) (2003). Dewey: The Collected Works of John Dewey, 1882-1953. (2nd Release). Electronic Edition. The Later Works of John Dewey, 1925-1953. Volume 16: 1949, Knowing and the Known, Essays, Typescripts, and Knowing and the Known, Carbondale, IL, Southern Illinois University Press.

Dewey, J., Boydston, J.A. (eds) (2008). Dewey: The Collected Works of John Dewey, 1882-1953. (2nd Release). Electronic Edition. The Later Works of John Dewey, 1925-1953. Volume 10: 1934, Art as Experience, Essays, Typescripts, and Knowing and the Known, Carbondale, IL, Southern Illinois University Press.

Elkjaer, B. (2004). Organizational Learning: the 'Third Way'. Management Learning, 35(4), 419-434. Doi : https://doi.org/10.1177/1350507604048271.

Elkjaer, B. (2017). Organizations as Real and Ephemeral. On Pragmatism and Learning as Briging Organization and Organizing. Zeitschrift für Weiterbildungsforschung, 40(1), 53-68. Doi : https://doi.org/10.1007/s40955-017-0086-0

Foucault, M. (1975). Surveiller et punir. Paris : Gallimard.

Gherardi, S. (2013). Is organizational learning possible without participation? Dans S. M. Weber, M. Göhlich, A. Schröer, Cl. Fahrenwald \& H. Macha (Eds), Organisation und Partizipation. Beiträge der Kommission Organisationspädagogik (pp. 2943). Wiesbaden: Springer.

Gillberg, C. \& Vo, L. C. (2011). Approche pragmatiste de la connaissance et de l'apprentissage dans les organisations. Management Avenir, 43(3), 410-427. Doi : https://doi.org/10.3917/mav.043.0410

Hjorth, D., Holt, R. \& Steyaert, C. (2015). Entrepreneurship and Process Studies. International Small Business Journal, 33(6), 599-611. Doi : https://doi. org/10.1177/0266242615583566

Jeanneret, Y. (2013). Faire trace : un dispositif de représentation du social. Intellectica, 59(1), 41-63.

Jolivet, A. \& Vasquez, C. (2011). Reconfiguration de l'organisation : suivre à la trace des figures textualisées - le cas de la figure du patient. Études de communication, 36, 129-146. Doi : https://doi.org/10.4000/edc.2563

Larroche, V. (2018). Le dispositif: un concept pour les sciences de l'information et de la communication. Tome 3. Coll. Science cognitive et management des connaissances. Londres : ISTE Group.

Lombardo, É. \& Angelini, C. (2013). Médiations mémorielles : le dispositif en tant que médiateur. Communication et organisation, 43, 193-204.

Lorino, P. (2007, July). Communautés d'enquête et création de connaissances dans l'organisation : le modèle de processus en gestion. Annales des télécommunications, 62(7), 753-771. Doi : https://doi.org/10.1007/BF03253288 
Lorino, P. (2013). L'activité collective, processus organisant : un processus discursif fondé sur le langage pragmatiste des habitudes. Activités, 10(10-1). Doi : https://doi. org/10.4000/activites.656

Lorino, P. (2018). Pragmatism and Organization Studies. Oxford: Oxford University Press.

Mair, J. \& Marti, I. (2006). Social Entrepreneurship Research: A Source of Explanation, Prediction, and Delight. Journal of World Business, 41(1), 36-44. Doi : https:// doi.org/10.1016/j.jwb.2005.09.002

Martela, F. (2015). Fallible Inquiry with Ethical Ends-in-view: A Pragmatist Philosophy of Science for Organizational Research. Organization Studies, 36(4), 537-563. Doi : https://doi.org/10.1177/0170840614559257

Meunier, J. P. (1999). Dispositif et théories de la communication. Deux concepts en rapport de codétermination. Hermès, 25(3), 83-91. Doi : https://doi. org/10.4267/2042/14976

O’Miel, J., Gourgues, G., Mazeaud, A., Nonjon, M. \& Parizet, R. (2017). Une sociologie critique de la démocratie participative est-elle utile ? Participations, 3, 221-242. Doi : https://doi.org/10.3917/parti.019.0221

Peeters, H. \& Charlier, P. (1999). Contributions à une théorie du dispositif. Hermès, 25(3), 15-23. Doi : https://doi.org/10.4267/2042/14969

Robichaud, D., Giroux, H. \& Taylor, J. R. (2004). The Metaconversation: The Recursive Property of Language as a Key to Organizing. Academy of Management Review, 29(4), 617-634. Doi : https://doi.org/10.5465/amr.2004.14497614

Roginsky, S. \& Christophe, C. (2018). Étudier l'hybridation organisationnelle par le prisme des publics : les publics comme pierre angulaire des tensions qui animent une association de solidarité internationale. Recherches en communication, 47, 131158. Doi : https://doi.org/10.14428/rec.v47i47.45573

Rupcic, N. (2020). Learning Organizations and Organizational Learning through the Pragmatist Lens. The Learning Organization, 27(5), 463-472. Doi : https://doi. org/10.1108/TLO-07-2020-258

Zask, J. (2008). Le public chez Dewey : une union sociale plurielle. Tracés. Revue de sciences humaines, 15, 169-189. Doi : https://doi.org/10.4000/traces.753

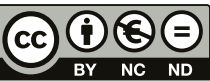
«Attibution - pas d'utilisation Commerciale - Pas de Modification 4.0. International» (CC BY-NC-ND) 